\title{
Economic Dynamic Real-Time \\ Optimization and Nonlinear Model-Predictive Control on Infinite Horizons
}

\author{
Lynn Würth* James B. Rawlings ${ }^{* *}$ Wolfgang Marquardt ${ }^{* * *}$ \\ * AVT - Process Systems Engineering, RWTH Aachen University, \\ D-52056 Aachen, Germany (email: lynn.wuerth@avt.rwth-aachen.de). \\ ** Dept. Chem. \& Biol. Eng. - Univ. of Wisconsin. Madison (WI), \\ USA (email: rawlings@engr.wisc.edu) \\ *** AVT - Process Systems Engineering, RWTH Aachen University, \\ D-52056 Aachen, Germany (email: \\ wolfgang.marquardt@avt.rwth-aachen.de).
}

\begin{abstract}
This paper investigates the formulation of nonlinear model-predictive control problems with economic objectives on an infinite horizon.

The proposed formulation guarantees nominal stability for closed-loop operation. Furthermore, a novel solution method of the infinite horizon method through a transformation of the independent time variable is employed. The closed-loop optimization with infinite horizon is compared to a finite-horizon formulation. A small case study is presented for illustration purposes.
\end{abstract}

Keywords: Dynamic Real-Time Optimization, Economic Objective, Nonlinear Model-Predictive Control, Infinite Horizon, Stability.

\section{INTRODUCTION}

The interest in economic Dynamic Real-Time Optimization (DRTO) or Nonlinear-Model Predictive Control (NMPC) with economic objectives has increased (Backx et al. (2000), Helbig et al. (2000), Engell (2007), Rawlings and Amrit (2008), Zavala (2008)), as the development of efficient methods for solving these types of optimization problems has significantly progressed in recent years. Compared to the traditional formulation of NMPC problems with quadratic cost criteria minimizing the deviation from a fixed steady-state set-point, the economic dynamic optimization problem exploits all the dynamic degrees of freedom available to maximize the profit of the plant on a given time horizon. Furthermore, the profit is maximized at a sampling rate of high frequency, whereas the traditional steady-state optimization is performed at a slow rate and only when the process is in a steady-state. Thus, disturbances with a favorable impact on the profit can also be exploited efficiently instead of compensating them by minimizing a steady-state offset.

However, theoretical studies of DRTO and NMPC problems with economic objectives are still lacking. Huesman et al. (2008) have pointed out that certain formulations with linear economic objective functions lead to multiple solutions. They concluded that some degrees of freedom are left for optimization. These degrees of freedom can be exploited by introducing a second optimization problem to improve the operability.

Rawlings and Amrit (2008) have shown that in order to optimize process economics it is sometimes advanta- geous not to reach the steady-state quickly. Secondly, they pointed out that the formulation with an economic objective results in characteristic trajectories behaving like a turnpike. The trajectory is attracted to a constant path and finally moves away from the constant path at the end of the horizon. The turnpike is a characteristic property of the economic optimization problem with finite-horizon, which has been introduced and studied in the economics literature before (Carlson et al., 1991).

In the economics literature an infinite horizon formulation is often employed. Infinite horizons have also been considered in the MPC literature as stabilizing although impractical for online application. A review of methods providing stability but circumventing infinite horizons by adding a terminal constraint, a terminal cost function, or by employing a terminal constraint set with a local stabilizing controller have been reviewed by Mayne et al. (2000).

An infinite-horizon formulation for (nonlinear) economic dynamic optimization on a receding horizon is explored in this paper. There are several advantages related to an infinite horizon formulation:

(1) The somewhat arbitrary choice of the final time of the optimization horizon is avoided. A natural formulation of the optimization problem is achieved for continuous processes if the final time is not specified.

(2) The infinite-time horizon formulation leads to closedloop operation with guaranteed stability. This property was also exploited in the literature of linear MPC, where the infinite-horizon formulation can 
be transformed into a finite horizon formulation by adding a terminal term to the cost functional (Muske and Rawlings, 1993).

In the following, the formulation of the economic optimization problem will be investigated and a comparison between finite and infinite horizon problems will be carried out. A new approach is presented for solving the infinitehorizon problem numerically. We use a transformation of the infinite-time onto a finite-time horizon and combine this transformation with an adaptive discretization. As already noted in the literature investigating stability in MPC, very long horizons provide stable control but lead to high computational costs. Many solution methods employed in MPC use a discretization of the control variables with a uniform spacing of grid points. An alternative adaptive discretization (Schlegel et al., 2005) introduces grid points mainly within the transient parts of the profile and reduces the computational load. Note that this method can also be applied to solve the infinite-horizon problem in the MPC regulator case to track a given set-point.

\section{ECONOMIC DRTO PROBLEM}

\subsection{Finite-Horizon Formulation}

The moving horizon formulation of the DRTO problem is similar to the formulation used in nonlinear modelpredictive control, although an economic objective is chosen to provide an economically optimal operation at all times (Helbig et al., 2000). The moving horizon problem is defined as follows:

$$
\begin{gathered}
\min _{\boldsymbol{u}^{j}(t)} \Phi\left(\boldsymbol{x}, \boldsymbol{u}, t_{0}, t_{f}\right) \\
\text { s.t. } \quad \dot{\boldsymbol{x}}(t)=\boldsymbol{f}\left(\boldsymbol{x}(t), \boldsymbol{u}^{j}(t)\right), \\
\boldsymbol{y}(t)=\boldsymbol{g}\left(\boldsymbol{x}(t), \boldsymbol{u}^{j}(t)\right), \\
\boldsymbol{x}\left(t^{j}\right)=\hat{\boldsymbol{x}}^{j}, \\
\mathbf{0} \geq \boldsymbol{h}\left(\boldsymbol{x}(t), \boldsymbol{y}(t), \boldsymbol{u}^{j}(t)\right), \\
\mathbf{0} \geq \boldsymbol{e}\left(\boldsymbol{x}\left(t_{f}^{j}\right)\right), \\
t \in\left[t^{j}, t_{f}^{j}\right], \\
t^{j}:=t^{j-1}+\Delta t, \\
j=0,1, \ldots J .
\end{gathered}
$$

$\boldsymbol{x}(t) \in \mathbb{R}^{n_{x}}$ are state variables with initial conditions $\hat{\boldsymbol{x}}^{j}$; $\boldsymbol{y}(t) \in \mathbb{R}^{n_{y}}$ are algebraic output variables. The dynamic process model (2) is given by $\boldsymbol{f}(\cdot)$. The time-dependent control variables $\boldsymbol{u}(t) \in \mathbb{R}^{n_{u}}$ are degrees of freedom for the optimization problem. The optimization problem is solved on the time horizon $\left[t^{j}, t_{f}^{j}\right]$ at each sampling instant $t^{j}$; the control is implemented on the current sampling interval (assuming negligible computational time), and the optimization horizon is then shifted by the sampling interval $\Delta t$. Equations (5) and (6) describe the path constraints $\boldsymbol{h}(\cdot)$ on the input and state variables and the endpoint constraints $\boldsymbol{e}(\cdot)$ on the state variables. Process operation is determined by economic decision criteria, which enter into the definition of the objective function $\Phi(\cdot)$. Exemplarily the profit function can be defined as

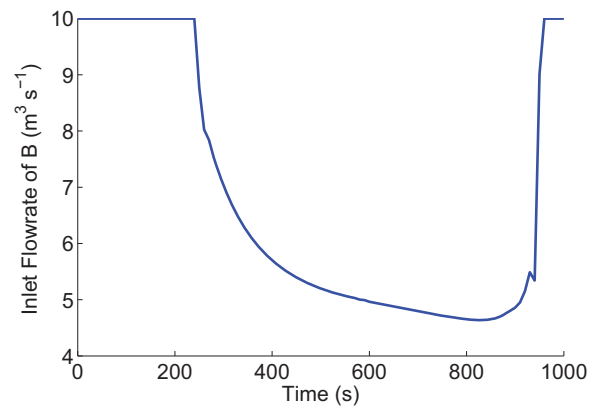

(a) Final time of $1000 \mathrm{~s}$

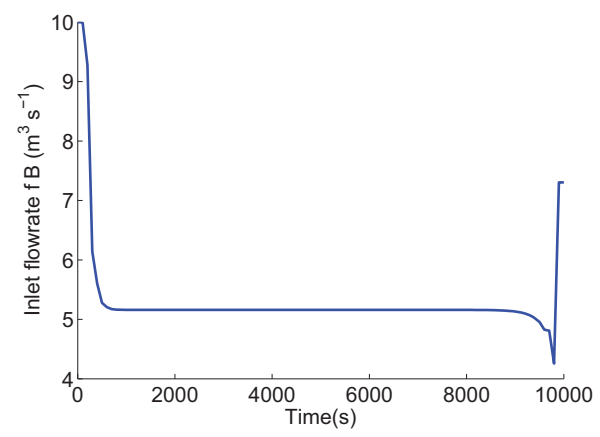

(b) Final time of $10000 \mathrm{~s}$

Fig. 1. Finite horizon

$$
\Phi=-\int_{0}^{T}\left(c_{\text {prod }} \dot{n}_{\text {prod }}-c_{\text {reac }} \dot{n}_{\text {reac }}-\dot{q}\right) d t
$$

where $c_{\text {prod }}$ and $c_{\text {reac }}$ are the costs of the products and reactants, and $\dot{n}_{\text {prod }}$ and $\dot{n}_{\text {reac }}$ are the flowrates of the products and reactants. The term $\dot{q}$ includes utility costs, depreciation, and other expenses.

If we choose a finite horizon in a dynamic optimization problem with economic objective, we often observe a socalled turnpike effect when looking at the solution profile (Rawlings and Amrit (2008), Carlson et al. (1991)). The turnpike effect means that the trajectory spends most time at a balanced equilibrium path, which is independent of the initial condition and the final time. Under certain conditions, this turnpike reduces to a singleton. In that case, the characteristic behavior is that the trajectory is attracted by a stationary path, at a certain time $t_{m}$ and stays on this constant path, until it reaches a point $t_{n}$ close to the end of the horizon and moves away from the path at the end of the horizon. This gives rise to trajectories as shown in Figure 1. The trajectories represent the inlet flowrate of the reactant of the CSTR presented in Section 5 ; they were computed for different final times. In these figures the solution path strongly depends on the choice of the final time. In the first figure, the final time is not long enough to let the process reach the turnpike. However, if a long horizon is chosen, the process gets on a constant path $\bar{k}$ (Carlson et al., 1991). The optimal steady-state path represents an attractor for the finite horizon path.

In practice, often a long horizon length is chosen to achieve closed-loop stability (Mayne et al., 2000). For stable plants, the final time $T$ is often chosen to be large compared to the settling time of the plant. The somewhat arbitrary choice of the final time suggests that more 
research is required to reconsider the current formulations and to look for adequate formulations of economic dynamic optimization problems providing stability in closed loop.

\subsection{Infinite Horizon Formulation}

For a given optimal trajectory, according to Bellmann's optimality principle, the trajectory starting from any point on the optimal trajectory is optimal for the corresponding problem initiated at that point on the trajectory. This implies that the formulation of the optimization problem on an infinite horizon is providing stability in closed loop.

If the profit function is optimized on a long or an infinite horizon, the time value of money should be accounted for. This is accomplished through the parameter $\rho$ discounting the future profit to the present value. If we deal with discrete payment periods, discounting the future amounts to today's value can be included in the calculation of the net present value. The objective function maximizing the net present value of cash flows $C_{k}$ in $N$ discrete time periods and with discounting rate $\rho$ is formulated as

$$
\Phi=\sum_{k=0}^{N} \frac{C_{k}}{1+\rho^{k}}
$$

where $k$ is the index of the time period. In this work we investigate an objective function with continuous discounting on an infinite horizon:

$$
\Phi=-\int_{0}^{\infty} e^{-\rho t}\left(c_{\text {prod }} \dot{n}_{\text {prod }}-c_{\text {reac }} \dot{n}_{\text {reac }}-\dot{q}\right) d t .
$$

The exponential formulation is usually employed for optimal control problems in continuous time and is equivalent to the discrete-time formulation in equation (11). Both formulations use, however, the same discount factor $\rho$, which can be chosen as the annual market interest rate.

Note that researchers in macroeconomics have included infinite horizons in their problem formulations very early to model e.g. economic growth (Barro and Sala-i-Martin, 1995). This is due to the fact that there is no natural finite time in these types of problems and the consequences of investment are very long-lived. Since this theory is not well-known in the systems and control community, an example of an economic growth model by Cass (1966) is shown here for illustration of the economic growth problem formulation:

$$
\begin{aligned}
y(t) & =f(k(t)) \\
c(t) & =y(t)-z(t) \\
\dot{k}(t) & =z(t)-\mu k(t) \\
k(0) & =k_{0}
\end{aligned}
$$

$k(t)$ is the stock of capital accumulated at time $t$. The production function $f(\cdot)$ associates an output $y$ with the capital stock $k$. The output $y(t)$ can be either consumed at a rate $c(t)$ or invested at a rate $z(t)$. The capital stock depreciates at a constant rate $\mu$. Cass (1966) considered the welfare functional

$$
W=\int_{0}^{T} e^{-\rho t} U(c(t)) d t
$$

where $U(c)$ is the concave utility function depending on the level of consumption $c(t)$. The maximization of $W$ is the standard optimal control problem and the final time $T$ is often assumed to be infinite in economic growth theory. If the discount factor $\rho$ is strictly positive, the objective function on an infinite horizon is bounded. However, if the discount factor is zero, the objective function becomes unbounded. Methods for reducing the unbounded objective on an infinite horizon to finite rewards have been presented by Carlson et al. (1991). We consider in this paper the case where the discount factor is positive, as it seems to be a reasonable assumption to include the time value of money on a long or infinite-time horizon. The discount factor is chosen in the range of the interest rate of the market.

Very few numerical solution methods exist in the literature of mathematical economics to solve the infinitetime horizon problem. Often, the indirect methods of optimal control are used to derive the First-Order Necessary Conditions of Optimality and a two-point boundary-value problem has to be solved. In the following, we will apply a numerical solution method with adaptive grid refinement to solve nonlinear infinite-horizon problems.

\section{INFINITE HORIZON SOLUTION APPROACH}

Solving the infinite-horizon problem is not straightforward and several attempts approximating the infinite horizon with finite horizons or reformulating the infinite-time problem exist in the literature.

\subsection{Time Transformation}

A common approach replaces the infinite-time horizon by a finite-time horizon, thereby introducing a truncation error. A second possibility is to use a variable transformation to transform the infinite-horizon into a finite-horizon problem. We choose the second approach in this work, since it does not introduce truncation errors and allows to obtain a solution of higher accuracy of the optimization solution on the infinite horizon.

The infinite horizon with $t \in[0, \infty)$ can be transformed into a finite horizon with $\tau \in[0,1]$ using the variable transformation

$$
\tau=t /(1+t)
$$

of the independent variable $t$. A similar variable transformation was presented by Kunkel and Hagen (2000) to obtain solutions of the infinite-horizon optimal control problem. The variable transformation of eq. (2) yields the transformed system:

$$
\frac{d \boldsymbol{x}}{d \tau}=\frac{\boldsymbol{f}(\boldsymbol{x}(\tau), \boldsymbol{u}(\tau))}{(1-\tau)^{2}}
$$

We can see that a singularity is introduced at $\tau=1$, which corresponds to $t=\infty$.

In order to study the singularity at $\tau=1$, we will restrict the analysis to the scalar system

$$
\frac{d x}{d \tau}=\frac{f(x(\tau))}{(1-\tau)^{2}}
$$

The eigenvalue problem corresponding to eq. (19) is

$$
\frac{d x}{d \tau}=\frac{\lambda x}{(1-\tau)^{2}} \text {. }
$$

The general solution of this differential equation is

$$
x(\tau)=c e^{\frac{\lambda}{1-\tau}} .
$$


If we assume that the system is within the region of attraction of a stable steady-state, we obtain the limit

$$
\lim _{\tau \rightarrow 1} c e^{\frac{\lambda}{1-\tau}}=0
$$

if $\lambda<0$ and the system will reach steady-state as $\tau \rightarrow 1$.

However, if $\lambda>0$, the system is unstable, and

$$
\lim _{\tau \rightarrow 1} c e^{\frac{\lambda}{1-\tau}}=\infty \text {. }
$$

In that case we have to handle the singularity at $\tau=1$ by imposing a boundary condition at final time. The steady-state solution can be imposed as terminal boundary condition. If we are dealing with an unstable system, a numerical solution approach different from the singleshooting method presented in the following section must be employed. The boundary value problem problem could be solved using multiple-shooting or orthogonal collocation methods. In order to generalize the analysis of the singularity above, an extension to the multivariable case should be performed including an analysis of the stable and unstable modes of the system.

\subsection{Adaptive Discretization}

The continuous control variables in optimization problem (1) are discretized after the time transformation with eq. (18) using piecewise-constant or piece-wise linear approximations. The discretization and the formulation of the NLP for the piecewise constant approximation reads as

$$
\mathbf{u}_{i}\left(\tau_{k}\right)=\mathbf{c}_{\mathbf{u}_{i, k}}, k=1, \ldots, N, i=1, \ldots, n_{u},
$$

where $N$ is the number of discretization intervals and $n_{u}$ the number of control variables. Choosing the discretized controls $\mathbf{z}_{i}:=\left[\mathbf{c}_{\mathbf{u}_{i, k}}\right]$, as the $n_{z}$ optimization variables, the dynamic optimization problem can be transcribed into the NLP

$$
\begin{gathered}
\min _{\boldsymbol{z}} f(\boldsymbol{z}):=\Phi(\boldsymbol{z}) \\
\text { s.t. } \boldsymbol{g}(\boldsymbol{z}) \geq 0 .
\end{gathered}
$$

The nonlinear program can be solved by employing a standard SQP algorithm. Since the optimization algorithm requires repetitive function evaluations and gradients, the objective function $\Phi$, constraints $\mathbf{g}(\cdot)$ and their gradients are evaluated by a simultaneous integration of the process model and the sensitivity equation system.

The optimization problem is solved using the dynamic optimization software DyOS (2002), which adopts an adaptive control vector parameterization (Schlegel et al., 2005). The adaptation is done using a refinement method based on a wavelet analysis of the control profile. If the horizon of the optimization problem is infinite or has a long finite time, an adaptive discretization is essential to deal with the computational load associated with these long or infinite horizons. The adaptive discretization allows to introduce the grid points selectively in transient regions of the control profile, and therefore avoids overloading the optimization problem with many optimization parameters. Furthermore, a highly accurate solution profile can be obtained.

\subsection{Closed-loop Implementation}

The solution approach outlined in the previous section is implemented in an algorithm for closed-loop dynamic optimization. The optimization is performed on the transformed timehorizon $[0,1]$, and the results are converted back to the original timehorizon.

for $j=1, N$ (number of sample intervals) do

(1) Solve the optimization problem (26), where the control variables $\boldsymbol{u}^{j}(\tau)$ have been discretized on $[0,1]$, to obtain the solution $\boldsymbol{z}^{j}$.

(2) Transform the control variables $\boldsymbol{u}^{j}(\tau), \tau \in[0,1]$ back to the original representation $\boldsymbol{u}^{j}(t), t \in[0, \infty)$.

(3) Implement the control variables $\boldsymbol{u}^{j}(t)$ for one sampling interval $\left[t_{j}, t_{j+1}\right]$. Get measurements and compute state estimates.

(4) Horizon shift: Reduce the time horizon by one sampling interval and use the shifted solution as initial guess for the next optimization problem.

(5) Transform the shifted variables $\boldsymbol{u}^{j}(t)$ to the finite horizon representation $\boldsymbol{u}^{j}(\tau), \tau \in[0,1]$ using eq. (18).

end for

\section{INFINITE-HORIZON NMPC}

Apart from the economic dynamic optimization problem, the presented solution method for infinite-horizon formulations can also be used for the NMPC regulator problem, if we deal with a pure tracking problem. In that case the economic objective function is replaced by the quadratic objective function

$$
\Phi=\int_{0}^{\infty}\left(\Delta \boldsymbol{u}^{T} \boldsymbol{Q} \Delta \boldsymbol{u}+\left(\boldsymbol{y}^{\text {set }}-\boldsymbol{y}\right)^{T} \boldsymbol{W}\left(\boldsymbol{y}^{\text {set }}-\boldsymbol{y}\right)\right) d t
$$

minimizing the deviation from fixed set-points $\boldsymbol{y}^{\text {set }}$ and the control moves $\Delta \boldsymbol{u}$. As a finite horizon is traditionally used in NMPC, some methods guarantee closed-loop stability by introducing e.g. a terminal constraint. However, introducing a terminal constraint can lead to feasibility problems. By employing the infinite-horizon solution method, the disadvantages of the finite-horizon methods to guarantee closed-loop stability can be avoided.

\section{CASE STUDY}

The approach is applied to the benchmark case of the Williams-Otto continuous stirred tank reactor, as introduced by Forbes (1994). The reactions taking place in the reactor are

$A+B \stackrel{k_{1}}{\longrightarrow} C, C+B \stackrel{k_{2}}{\longrightarrow} P+E, P+C \stackrel{k_{3}}{\longrightarrow} G$.

Reactant $\mathrm{A}$ is already present in the reactor, whereas reactant $\mathrm{B}$ is fed continuously to the reactor. During the exothermic reactions the desired products $\mathrm{P}$ and $\mathrm{E}$ as well as the side-product $\mathrm{G}$ are formed. At the end of the batch, the conversion to the main products $\mathrm{P}$ and $\mathrm{E}$ should be maximized. During the batch, constraints on the inlet flow rate of reactant $\mathrm{B}\left(F_{B_{i n}}\right)$ and the reactor temperature $\left(T_{r}\right)$ must be fulfilled. The manipulated control variables of this process are $F_{B_{i n}}$ and $T_{r}$. The CSTR is assumed to be well-mixed and the dynamics of the cooling system are neglected. The reactor system is open-loop stable and therefore the singularity issues at $\tau=1$ do not arise in this case study. 

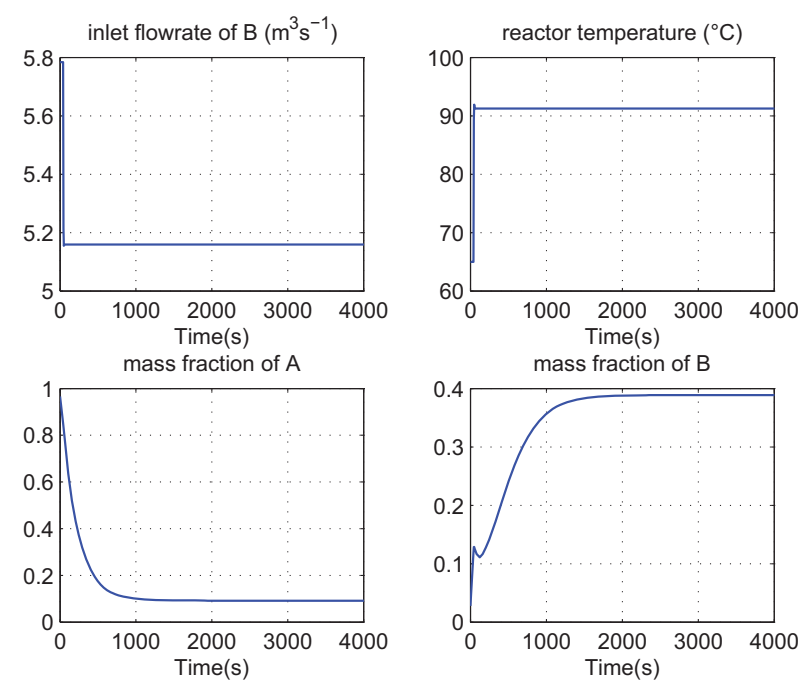

(a) Infinite horizon, open-loop
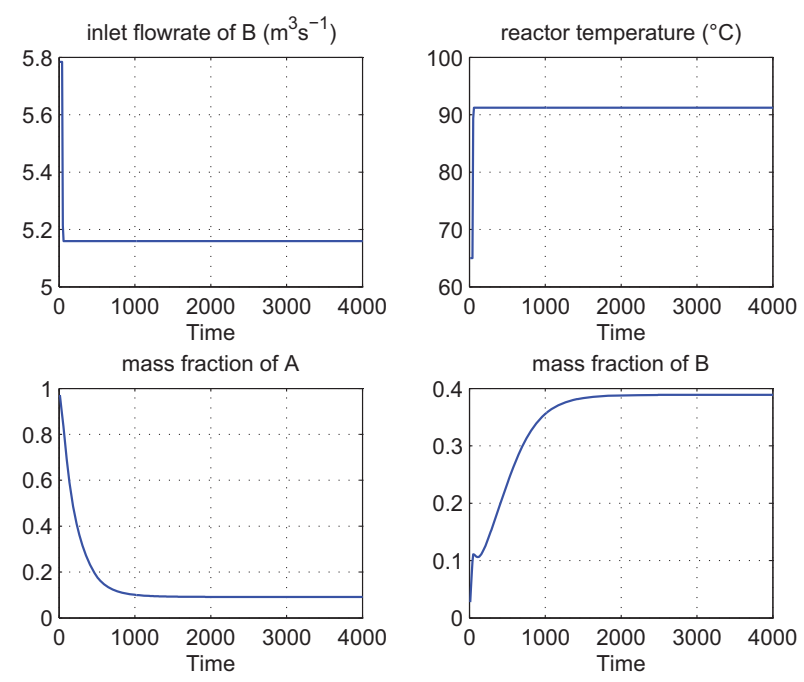

(b) Infinite horizon, closed-loop

Fig. 2. Infinite horizon results

\subsection{Economic DRTO Problem}

The economic objective is to maximize the profit, which consists of the revenue obtained from the products minus the costs of the reactants over the infinite time horizon. Furthermore, an annual discount rate of $5 \%$ is chosen to account for the time value of money. The optimization problem is formulated as follows:

$\max _{F_{B_{i n}}(t), T_{r}(t)} \Phi=\int_{0}^{\infty} e^{-\rho t}\left(c_{p} \dot{n}_{p}+c_{e} \dot{n}_{e}-c_{a} \dot{n}_{a}-c_{b} \dot{n}_{b}\right) d t$

s.t. process model, and

$0 \frac{\mathrm{kg}}{\mathrm{sec}} \leq F_{B_{i n}}(t) \leq 5.784 \frac{\mathrm{kg}}{\mathrm{sec}}$,

$0{ }^{\circ} \mathrm{C} \leq T_{r}(t) \leq 150{ }^{\circ} \mathrm{C}$.

\subsection{Closed-Loop Results}

The economic performance of the operation of the CSTR is optimized for both infinite and finite horizon formulations
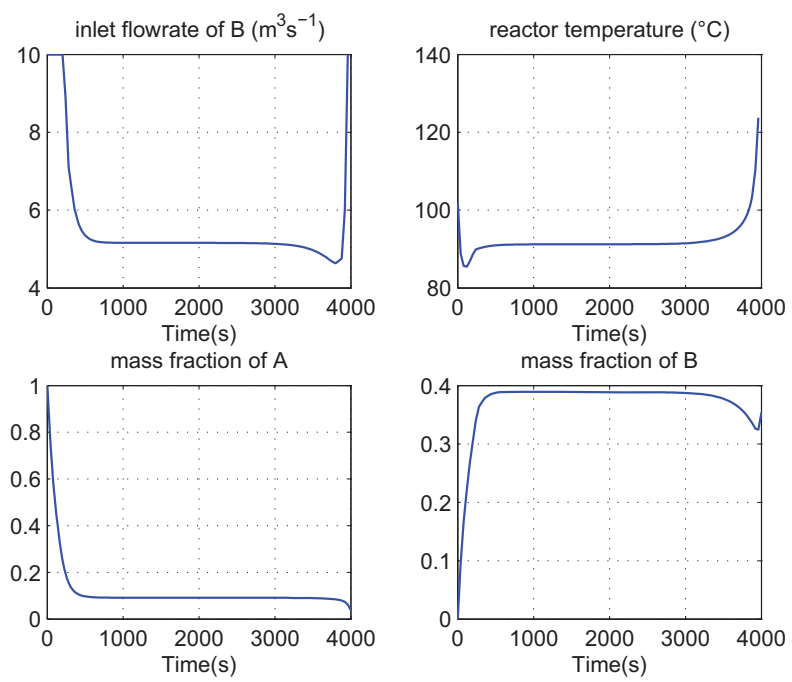

(a) Finite horizon, open-loop
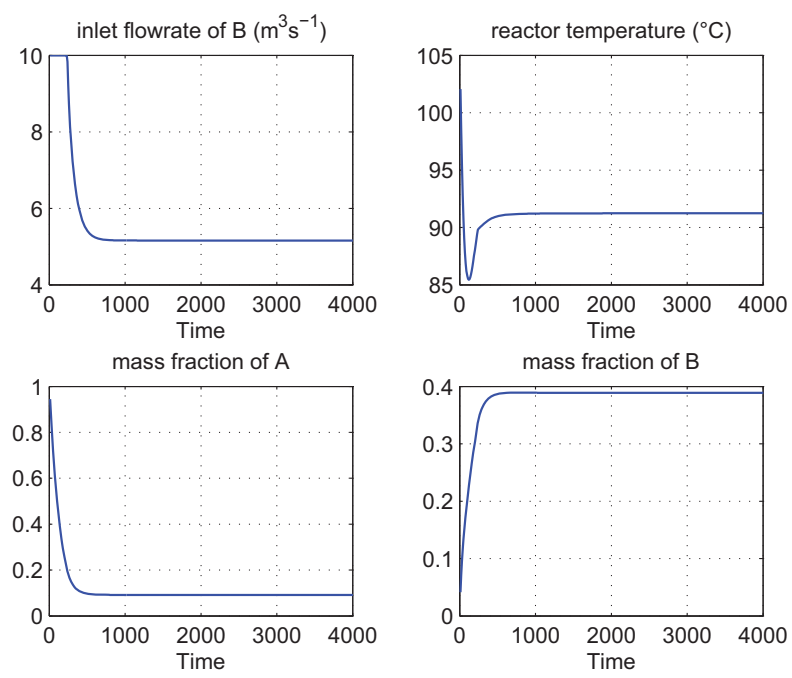

(b) Finite horizon, closed-loop

Fig. 3. Finite horizon results

on a receding horizon. The results for the infinite-horizon case are shown in Figure 2. The open-loop optimization problem is solved using the time transformation for $\tau$ $\in[0,1]$ and the results are simulated on a long horizon in the original time variable. The closed-loop results are obtained using the algorithm with adaptive grid refinement as sketched in Section 3.3. Figure 2 shows that the nominal trajectories in open-loop and closed-loop are almost identical except minor deviations due to the online adaptation scheme. This result confirms that the solution of the optimal problem on an infinite horizon in closed-loop is providing a nominally stable control with an economic objective. Furthermore, the computational effort has been low as only few degrees of freedom are required in the adaptive grid refinement approach. It is interesting to observe that the control variables reach their steady-state values very fast, but the state variables require more time to reach the steady-state values.

The results for the finite-horizon formulation with a final time of $4000 \mathrm{~s}$ are shown in Figure 3. In this case, the open-loop trajectory shows the turnpike behavior. Since 
only the first control interval is implemented in closedloop, the end of the trajectory in open-loop is actually never implemented. This leads to a discrepancy between the open-loop and the closed-loop behavior, as Bellmann's optimality principle is not fulfilled. Nevertheless, the figures show that the system reaches the steady-state quite fast.

Comparing the closed-loop results obtained with the infinite- and the finite-time horizon formulation, the figures show that the transient part of the trajectories at the beginning is quite different, but that the steady-states obtained after a certain time are identical. The temperature profile is different in the infinite-horizon (Figure 2.b) and the finite-horizon cases (3.b), as the temperature increases from $65^{\circ} \mathrm{C}$ to $90^{\circ} \mathrm{C}$ in (2.b) and the temperature decreases from $102^{\circ} \mathrm{C}$ to $91^{\circ} \mathrm{C}$ in (3.b). It was observed that this difference only occurs for relatively short horizons. If the final time chosen for the finite-horizon case increases, the trajectory of the finite-horizon case approximates the infinite-horizon case more closely. As expected, the longer the horizon becomes, the closer the solution of the finitehorizon problem will be to the infinite-time solution. This is due to the fact that the transient parts of the trajectories have less impact on the profit function for increasing length of the time horizon. As the same steady-state is reached by both finite- and infinite-time horizon formulations, the same profit is also obtained at steady-state.

These results show that also the finite-horizon formulation with economic objective can provide closed-loop stability, as the system gets on the turnpike (which corresponds to a stationary path in this case) and stays there for most of the time. Hence, by choosing a long time horizon it is possible to achieve closed-loop nominal stability, because the trajectory is attracted to the stationary path. However, as shown in Figure (1.a), if the finite time horizon is not long enough the resulting trajectories will not reach the optimal stationary path.

\section{CONCLUDING REMARKS}

The closed-loop solution of nonlinear DRTO or NMPC problems was studied. Nominal stability in closed-loop economic optimization is achieved via an infinite-horizon formulation. The comparison of the infinite- to the finitehorizon formulation shows that the formulation of DRTO problems on finite horizons can also provide closed-loop stability, if the optimization time horizon is chosen long enough such that the trajectory is attracted to a constant path.

Secondly, a new numerical approach was introduced for solving infinite-horizon problems addressed in NMPC and DRTO. The method achieves high computational accuracy because the infinite horizon is transformed into a finite horizon through a simple variable transformation. The advantage is that the truncation error occurring by choosing an arbitrary final time is avoided. Furthermore, the computational load is still low because of adaptive grid refinement resulting in low number of degrees of freedom for optimization.

In the future, it is of interest to further investigate the properties of the finite-horizon economic optimization problem required to achieve closed-loop stability. On the other hand, the infinite-horizon formulation with adaptive grid refinement is a promising approach to guarantee closed-loop stability. The solution method for infinitehorizon problems will be further developed and extended to open-loop unstable systems.

\section{REFERENCES}

Backx, T., Bosgra, O., and Marquardt, W. (2000). Integration of model-predictive control and optimization of processes. In IFAC Symposium "Advanced Control of Chemical Processes", ADCHEM 2000, 249-260. Pisa, Italy.

Barro, R. and Sala-i-Martin, X. (1995). Economic Growth. McGraw-Hill Advanced Series in Economics.

Carlson, D.A., Haurie, A.B., and Leizarowitz, A. (1991). Infinite Horizon Optimal Control. Springer Verlag.

Cass, D. (1966). Optimal growth in an aggregative model of capital accumulation, a turnpike theorem. Econometrica, 34, 833-850.

DyOS (2002). DyOS User Manual. Process Systems Engineering, Aachener Verfahrenstechnik, RWTH Aachen University.

Engell, S. (2007). Feedback control for optimal process operation. Journal of Process Control, 17, 203-219.

Forbes, J. (1994). Model Structure and Adjustable Parameter Selection for Operations Optimizations. Ph.D. thesis, McMaster University, Hamilton, Canada.

Helbig, A., Abel, O., and Marquardt, W. (2000). Structural concepts for optimization based control of transient processes. In F. Allgöwer and A. Zheng (eds.), Nonlinear Model Predictive Control, volume 26 of Progress in Systems and Control Theory, 295-311. Birkhäuser, Basel.

Huesman, A.E.M., Bosgra, O.H., and den Hof, P.M.J.V. (2008). Integrating MPC and RTO in the process industry by economic dynamic lexicographic optimization; an open-loop exploration. In Proceedings of the 2008 AICHE Annual Meeting. Philadelphia, USA.

Kunkel, P. and Hagen, O.V.D. (2000). Numerical solution of infinite-horizon optimal control problems. Computational Economics, 16(3).

Mayne, D.Q., Rawlings, J.B., RAO, C.V., and Scokaert, P.O.M. (2000). Constrained model predictive control: Stability and optimality. Automatica, 36(6), 789-814.

Muske, K.R. and Rawlings, J.B. (1993). Model predictive control with linear models. A.I.Ch.E. Journal, 39(2), $262-287$.

Rawlings, J.B. and Amrit, R. (2008). Optimizing process economic performance using model predictive control. In Assessment and Future Directions of Nonlinear Model Predictive Control. Pavia, Italy.

Schlegel, M., Stockmann, K., Binder, T., and Marquardt, W. (2005). Dynamic optimization using adaptive control vector parameterization. Comp. \& Chem. Eng., 29(8), 1731-1751.

Zavala, V. (2008). Computational Strategies for the Optimal Operation of Large-Scale Chemical Processes. Ph.D. thesis, Carnegie Mellon University. 